\title{
Assessing Effectiveness of an Audiovisual Educational Tool for Improving Dental Students' Probing Depth Consistency
}

Srividya Prabhu, Vanchit John, Steven Blanchard, George J. Eckert, Yusuke Hamada

Abstract: Dental students often underestimate their probing depth (PD) measurements, which emphasizes the need for effective and novel methods for teaching proper probing technique. The aim of this study was to evaluate the efficacy of audiovisual learning aids, recorded from the point of view of examiners, for improvement in PD agreement in dental students. In 2017-18, 22 thirdyear dental students were randomized into test and control groups. Each student and a single blinded faculty examiner performed PD measurements on a minimum of three patients. The test group viewed a video demonstrating proper probing technique, while the control group received only probing technique instruction from prior lectures. All measurements, the periodontal diagnoses, and the total time taken to complete PD measurements were recorded. A survey of student attitudes about the audiovisual tool was conducted after the intervention; all 22 students completed the survey. A total of 11,426 PD sites were measured. The test group had $10 \%$ greater accuracy in PD sites $=4 \mathrm{~mm}$. The control group had a minor but statistically significant increase in accuracy for $2 \mathrm{~mm}$ PD sites. For all incorrect measurements at sites PD $\geq 4$, the students tended to underestimate the PD. Tooth type, site location around tooth, and diagnosis had no significant effect on PD measurement agreement. No significant difference between groups was found for the proportions of gingivitis and periodontitis patients or for examination time. This study found that use of the audiovisual learning aid "Calibrated Periodontal Training Video" improved the students' probing depth accuracy for sites with PD of $4 \mathrm{~mm}$.

\begin{abstract}
Srividya Prabhu, DMD, MSD, is in private practice in Mason, OH; Vanchit John, BDS, MDS, DDS, MSD, is Chair, Department of Periodontics and Allied Dental Programs, Indiana University School of Dentistry; Steven B. Blanchard, DDS, MS, is Director of Graduate Periodontics, Indiana University School of Dentistry; George J. Eckert, MAS, is Biostatistician Supervisor, Department of Biostatistics, Indiana University School of Medicine; and Yusuke Hamada, DDS, MSD, is Associate Program Director, Department of Periodontics and Allied Dental Programs, Indiana University School of Dentistry. Direct correspondence to Dr. Yusuke Hamada, Department of Periodontics and Allied Dental Programs, Indiana University School of Dentistry, 1121 West Michigan Street, Indianapolis, IN 46202; 317-274-5121; yuhamada@iupui.edu.
\end{abstract}

Keywords: dental education, periodontics, periodontal disease, clinical skills, psychomotor skills, educational technology, instructional materials/methods, audiovisual, video, probing depth

Submitted for publication 6/14/18; accepted 9/18/18; first published online 2/11/19 doi: $10.21815 / J D E .019 .052$

$\mathrm{P}$ eriodontal disease is a chronic destructive inflammatory condition of the soft tissue and bony support of teeth affecting nearly $46 \%$ of the U.S. adult population. ${ }^{1}$ A comprehensive clinical and radiographic evaluation is necessary for formulating an accurate periodontal diagnosis and suitable treatment plan for patient management. While the 1999 Classification of Periodontal Diseases and Conditions provided a diagnosis scheme based on clinical attachment loss calculations alone, the updated 2017 World Workshop Classification suggests additional diagnostic guidelines for determining periodontal disease severity. ${ }^{2,3}$ Among these are periodontal probing depths (PDs), which are an easily obtained and useful diagnostic tool for acquiring clinical information regarding gingival health.
In the update, PDs $\leq 3 \mathrm{~mm}$ are associated with an intact periodontium/non-periodontitis patient, $4 \mathrm{~mm}$ are associated with Stage 1 periodontitis, $5 \mathrm{~mm}$ are associated with Stage 2, and $\geq 6 \mathrm{~mm}$ with Stage 3 . Based on these parameters, accurately detecting PDs $\geq 4 \mathrm{~mm}$ may be critical for recognizing a periodontal disease condition. ${ }^{3}$

Unfortunately, most patients with untreated periodontitis have periodontal inflammation and local etiologic factors that can affect probing and clinical attachment level measurement accuracy. ${ }^{4}$ Additional site-related factors may include level of disease severity, presence of plaque and/or calculus, root anatomy, and irregularities due to restorations. Accuracy may also be affected by clinician-dependent factors such as examiner experience, probing 
pressure, probing angulation, and visual assessment errors. ${ }^{5}$ These clinician-dependent factors are magnified in dental school settings. As confirmed in previous studies, it takes considerable time and experience for dental students to learn how to probe with correct angulation and technique within the sulcus. ${ }^{5,6}$ During this learning period, students are more prone to PD errors. Undetected probing errors in clinical settings can lead to the formulation of an incorrect diagnosis. As dental treatment decisions are contingent on periodontal status of a patient's dentition, probing discrepancies can ultimately lead to incorrect therapy and further problems. As emphasized by Drucker et al.'s study, more preclinical education and new teaching methods are needed for learning probing technique and improving accuracy of PDs in clinical settings. ${ }^{5}$

We have observed that, in preclinical didactic courses, periodontal examination skills are usually taught via diagrams and verbal explanations during traditional lectures or in lab settings. To our knowledge, no instruction in dental schools' preclinical education is done using audiovisual techniques from the instructor's point of view to demonstrate probing angulation, proper measurement points, methods of exploring sulcus with a probe, and appropriate mirror use to obtain optimal visualizations. Using audiovisual aids for periodontal examinations made by experienced periodontists may be an effective tool to improve students' comprehension and accuracy of probing depth measurements. The aim of this study was to evaluate the efficacy of audiovisual learning aids, recorded from the point of view of examiners, for improvement in PD agreement in dental students. We also sought to assess the effectiveness of educational audiovisual aids in improving PD discrepancies in periodontitis patients compared to gingivitis patients and in deeper PDs ( $\geq 4 \mathrm{~mm}$ ) compared to shallow pockets (1-3 $\mathrm{mm}$ pockets). We hypothesized that the test group would have significantly greater accuracy in their PDs than the control group.

\section{Materials and Methods}

Approval from the Indiana University Institutional Review Board was obtained (\#1706929900) for this quantitative, randomized controlled trial conducted in 2017-18. Prior to subject recruitment, a board-certified periodontist (coauthor $\mathrm{YH}$ ) conducted and recorded full mouth PD measurements on a volunteer with gingivitis with a camera mounted on loupes (Loupecam, 3x magnification, product distributed by VizVOCUS Inc., Scottsdale, AZ, USA; www.loupecam.com/), which allowed for audio and video recording from the point of view of the clinician. The recorded video was evaluated by two experienced, board-certified periodontists (coauthors $\mathrm{SB}$ and $\mathrm{VJ}$ ) to assess if the following was demonstrated correctly: probe angulation, measurement points, and "walking" technique. The examination recording was repeated until all three periodontists agreed with the probing methods. Once approved, the final audiovideo recording was edited using iMovie editing software (Apple Inc., Cupertino, CA, USA) to produce the "Calibrated Periodontal Training Video."

A recruitment email was sent to all 114 students in the third-year predoctoral class (D3) of the Indiana University School of Dentistry. Students who responded to the recruitment email were equally randomized into two groups (test and control groups) by alternating assignment based on the order of received email responses.

Separate preliminary instructional meetings were held for the test and control groups. Each participant was verbally questioned to confirm the number of months he or she had spent in predoctoral clinic and if he or she had any previous clinical experience prior to entering the predoctoral clinic. Participants were excluded if they reported having more than one year of clinical experience or any significant clinical experiences outside of the predoctoral clinic. Slideshow presentations were given at each meeting to review the steps of the study.

Each subject was asked to perform a periodontal examination (which includes PD measurements at six sites per tooth: the mesio-buccal, mid-buccal, disto-buccal, disto-palatal/lingual, mid-lingual/ palatal, and mesio-palatal/lingual) and to provide a periodontal diagnosis for a minimum of three of his or her own upcoming patients as part of the study. Patients could be included if they presented with a minimum of 20 teeth and no more than two missing teeth per quadrant (excluding third molars). Patients were to be excluded if they presented with limited mouth opening or a displaced tooth that prevented accurate measurement of PDs at all six measurement points per tooth. All participants were provided with group-specific instruction sheets outlining the steps of the patient examinations and checklists outlining patient inclusion and exclusion criteria.

The instruction provided at each preliminary meeting was identical except the "Calibrated Periodontal Training Video" was presented to the test 
group students only. These students were instructed to watch the video twice in succession prior to any patient exams. The test group students were provided with invitation-only online access to the video for online viewing and/or download. The control group received no additional training or instruction regarding periodontal PD technique other than what they previously received in didactic courses.

The students contacted the principal investigator (SP) regarding any upcoming appointments for patients requiring periodontal charting and who met the inclusion and exclusion criteria. These criteria were confirmed by two of the investigators (SP and $\mathrm{YH})$ prior to the start of each periodontal examination. Each student was provided with a paper periodontal chart for PDs to be recorded by his or her assistant. The total time of each PD measurement exam was recorded. A blinded faculty examiner (coauthor YH) then conducted his own PD exam of each patient, and the examiner's PD values were transcribed in the patient's official record for further dental treatment. After every exam, all diagnoses and stopwatch times were recorded on the paper charts. Both the student's paper charts and the faculty examiner's charts were collected by the research coordinator and deidentified. At the conclusion of the patient examination phase, the control group students were shown the "Calibrated Periodontal Training Video." Test and control group subjects were then asked to complete anonymous post-intervention questionnaires regarding their attitudes about and opinions of the training video.

A power analysis determined that a sample size of 60 patients ( 30 patients per group, each measured by the examiner and one student dentist) was needed to provide $80 \%$ power to detect a $10 \%$ difference in the proportion of sites with clinically acceptable variance between the two study groups. The comparison between the two groups for a difference in the proportion of patients with gingivitis or periodontitis was made using a chi-square test. Comparisons between the two groups for differences in the percentage of sites with clinically acceptable variance for PD measurement agreement (measurements within $1 \mathrm{~mm}$ ) were made using generalized linear mixed models. Additional analyses explored whether the group effect was modified by tooth type, site location around the tooth, PD measurement by the faculty examiner, and patient type (gingivitis vs. periodontitis patients) by testing interactions of these factors with the group effect. PD examination times in the test and control groups were compared using ANOVA. To compare the times between the students and the faculty examiner, the ANOVA included a fixed effect for student or faculty. Student's t-test was used to examine the survey results.

\section{Results}

An initial group of 24 students were recruited for the study. One student dropped out, and one student was excluded after revealing previous experience and training as a dental hygienist. A final total of 22 students participated in the study, which was $19.3 \%$ of the total class invited to participate. Students were equally randomized into the test and control groups, with 11 each. No statistically significant difference in the proportions of periodontal diagnosis (gingivitis and periodontitis) was found between the test and control groups (Table 1). A total of 11,426 PD sites were measured. Figure 1 shows the probing depth distribution of these sites as measured by the faculty examiner.

For examination times, no statistically significant difference was found between test and control groups for amount of time taken to complete PDs $(\mathrm{p}=0.11$; Table 2$)$. However, the students' exam times were found to be significantly longer than the faculty examiner's times ( $\mathrm{p}=0.0002$ for control group, $\mathrm{p}<0.0001$ for test group).

Sites were considered to have clinically acceptable variance if measurements were $\pm 1 \mathrm{~mm}$ compared to the faculty examiner's measurements.

Table 1. Number of patient examinations and percentage of patients diagnosed with gingivitis and periodontitis, by control and test groups

\begin{tabular}{|c|c|c|c|c|c|c|c|}
\hline \multirow[b]{2}{*}{ Group } & \multicolumn{4}{|c|}{ Patient Examinations } & \multicolumn{2}{|c|}{ Patient Diagnosis } & \multirow[b]{2}{*}{ p-value } \\
\hline & $\mathrm{N}$ & Mean (SD) & Min & Max & Gingivitis & Periodontitis & \\
\hline Control $(\mathrm{N}=11)$ & 38 & $3.5(1.1)$ & 2 & 6 & $22(58 \%)$ & $16(42 \%)$ & 0.36 \\
\hline Test $(\mathrm{N}=11)$ & 32 & $3.1(0.9)$ & 1 & 5 & $16(47 \%)$ & $18(53 \%)$ & \\
\hline
\end{tabular}




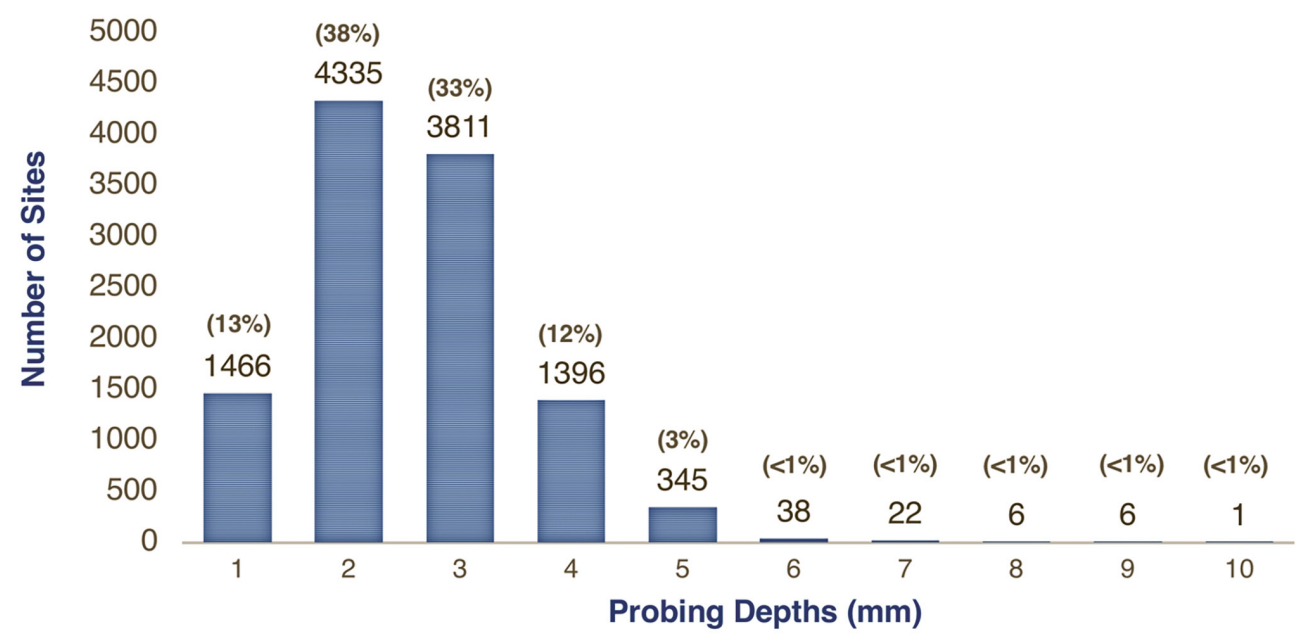

Figure 1. Number of sites by probing depth according to faculty examiner

Note: Percentages above bars are percentages of total number of sites $(N=11,426)$.

Table 2. Time (in seconds \pm SD) to complete probing depth examinations, by test and control groups

\begin{tabular}{|c|c|c|c|c|c|c|c|c|}
\hline Group & $\begin{array}{l}\text { Mean Exam } \\
\text { Time }\end{array}$ & $p$-value & Examiner & $\begin{array}{c}\text { Number of } \\
\text { Patient Exams }\end{array}$ & $\begin{array}{l}\text { Mean Exam } \\
\text { Time }\end{array}$ & Min & Max & p-value \\
\hline \multirow[t]{2}{*}{ Control } & $487 \pm 204$ & 0.11 & Faculty & 36 & $197 \pm 32$ & 127 & 291 & 0.0002 \\
\hline & & & Student & 36 & $485 \pm 209$ & 214 & 1100 & \\
\hline \multirow[t]{2}{*}{ Test } & $610 \pm 221$ & & Faculty & 32 & $199 \pm 41$ & 157 & 305 & $<0.0001$ \\
\hline & & & Student & 32 & $610 \pm 221$ & 302 & 1285 & \\
\hline
\end{tabular}

Note: Faculty examination times were inadvertently not recorded or incorrectly recorded during two control group patient exams, so these exams could not be included in the time data analysis.

There were no significant differences between the test and control groups for the percentage of sites with acceptable PD measurement variance (94\% with $95 \%$ Confidence Interval $=92 \%, 96 \%$ for test group; and $95 \%$ with $95 \%$ Confidence Interval $=93 \%, 96 \%$ for control group; $\mathrm{p}=0.74$ ).

Group comparison for percentage of sites with acceptable PD measurement variance was significantly affected by the PD measurement by the faculty examiner $(p=0.0002)$. For sites with $P D=2$ $\mathrm{mm}$, the control group had significantly greater accuracy ( $99 \%$ control vs. $97 \%$ test, $p=0.0158)$. For sites with $\mathrm{PD}=4 \mathrm{~mm}$, the test group had significantly greater accuracy than the control group (85\% test vs. $75 \%$ control, $\mathrm{p}=0.0056$; Figure 2 , panel A). No statistical differences were found regarding tooth type $(\mathrm{p}=0.24)$ or site location around tooth $(\mathrm{p}=0.68)$. Diagnosis $(\mathrm{p}=0.42)$ had no significant effect on group comparison nor between the test or control groups compared to the faculty examiner for $\mathrm{PD}$ sites $=1 \mathrm{~mm}$ $(\mathrm{p}=0.18), \mathrm{PD}=3 \mathrm{~mm}(\mathrm{p}=0.38), \mathrm{PD}=5 \mathrm{~mm}(\mathrm{p}=0.88)$, and $\mathrm{PD} \geq 6(\mathrm{p}=0.62)$. When we compared unacceptable student PD measurements against those of the faculty examiner, for all incorrect measurements at sites $\mathrm{PD} \geq 4$ there was a general tendency for students to underestimate the PD (panel B).

All 22 students completed the post-intervention survey. Students' attitudes about the efficacy of this audiovisual tool and preference for including in the curriculum were similar for the test and control groups (Table 3). Students showed the most interest in having audiovisual tools demonstrating extractions $(85.7 \%)$, surgical extractions (85.7\%), periodontal instrumentation and scaling and root planing (76.6\%), and suturing (76.2\%) (Figure 3 ).

\section{Discussion}

In this study, we hypothesized that the test group would have significantly more accuracy in their PDs compared to the control group. This hypothesis 
A)

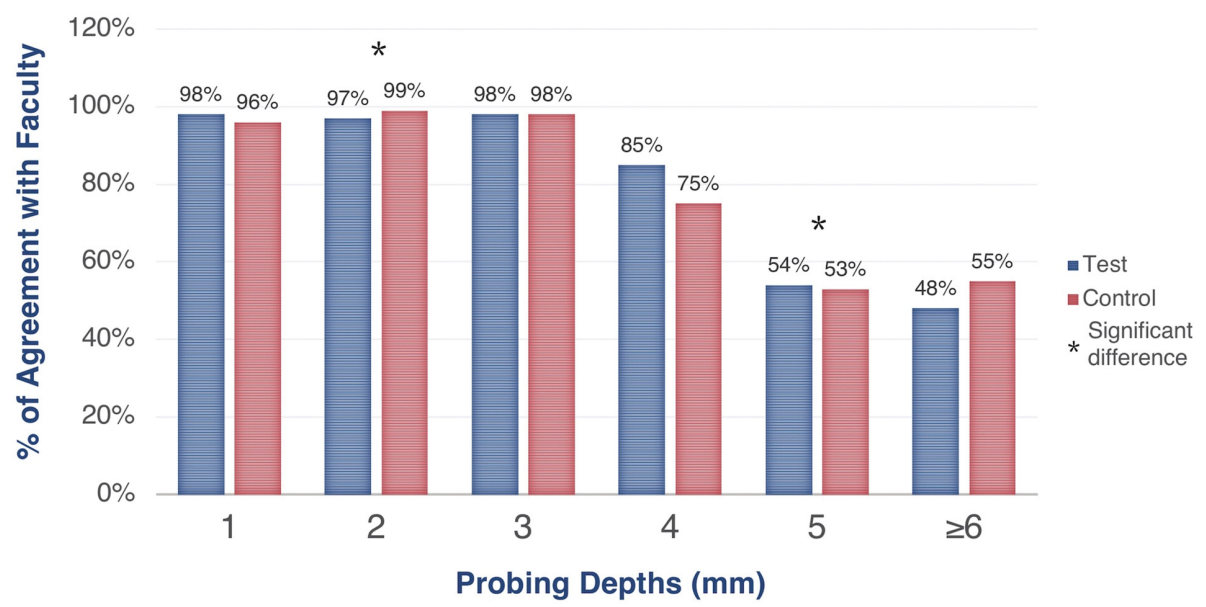

B)

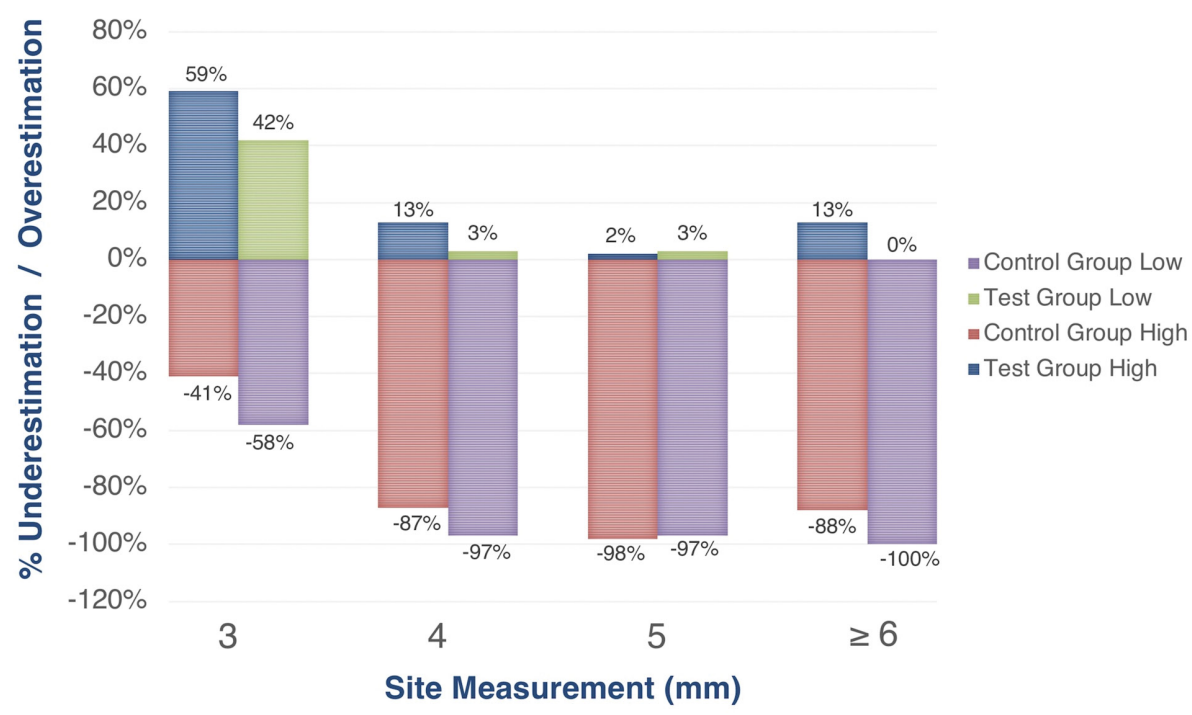

Figure 2. Test vs. control group comparison of probing depth accuracy by agreement with faculty assessment (panel A) and test vs. control groups' percentage of overestimation and underestimation (panel B)

Table 3. Students' survey responses about the audiovisual tool, by test and control groups: mean (SD)

\begin{tabular}{lcc} 
Group & $\begin{array}{c}\text { Helped in } \\
\text { Understanding } \\
\text { Periodontal Probing } \\
\text { Technique }\end{array}$ & $\begin{array}{c}\text { Should Be Part } \\
\text { of Clinical Skills } \\
\text { Curriculum }\end{array}$ \\
\hline Test & $4.6(0.5045)$ & $4.7(0.4671)$ \\
Control & $4.6(0.5164)$ & $4.7(0.4830)$
\end{tabular}

Note: Response options on both items ranged from $1=$ strongly disagree to $5=$ strongly agree. Full questions were as follows:

"This audiovisual aid helped my understanding of periodontal probing technique" (left column) and "Do you think this audiovisual aid should be available as part of your clinical skills curriculum?" (right column). was found to have limited validity. For sites measuring $4 \mathrm{~mm}$ in depth, the test group had $10 \%$ greater accuracy in measurements than the control group $(85 \%$ vs. $75 \%)$. This was a critical finding given the recent guideline provided by the 2017 World Workshop Classification, which established a $4 \mathrm{~mm}$ PD as the diagnostic cut-off for differentiating between an intact periodontium/non-periodontitis condition and Stage 1 periodontitis. ${ }^{3}$ Students who did not have access to the audiovisual teaching aid had incorrect PDs $25 \%$ of the time for $4 \mathrm{~mm}$ sites. No significant difference between the groups was found for PD accuracy for sites measuring $1,3,5$, and $>6 \mathrm{~mm}$ deep. It should be noted that there was a significantly lower 


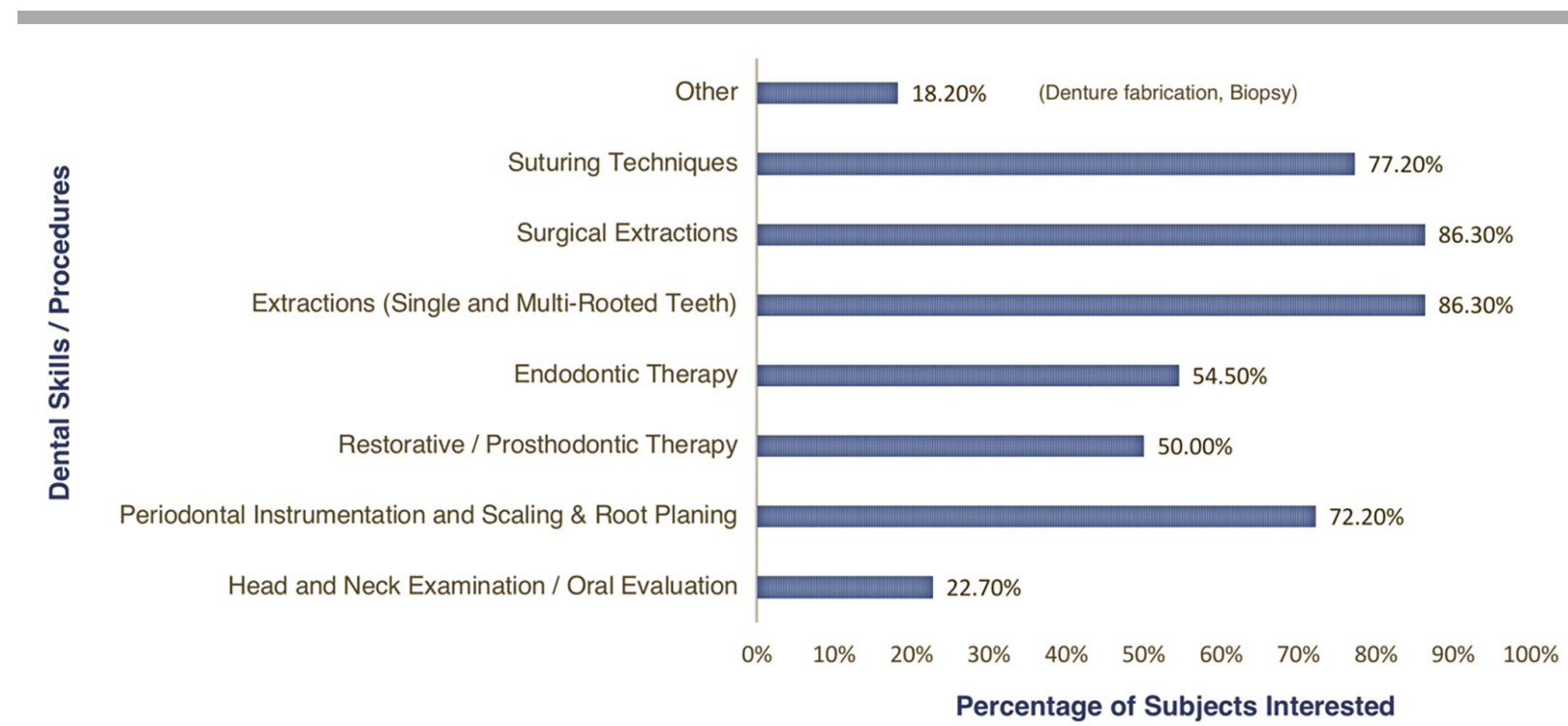

Figure 3. Students' interest in audiovisual tools on additional dental skills or procedures, by percentage of respondents $(\mathrm{N}=22)$

number of sites measuring $\geq 5 \mathrm{~mm}$ : only $3 \%$ of the total sites were $5 \mathrm{~mm}$ and $<0.5 \%$ of the sites were $>5 \mathrm{~mm}$. With so few of the sites probing $>4 \mathrm{~mm}$, it was difficult to detect any statistical differences in accuracy between the groups. Alternatively, the greater amount of PD disagreement in these deeper sites may indicate that, for sites $\geq 5 \mathrm{~mm}$, students had poorer accuracy, irrespective of instructional method.

Tooth type and site location around the tooth were found to have no significant effect on PD measurement agreement. These results contrast with those of Drucker et al., who reported higher discrepancy areas specifically in posterior and interproximal sites in their in vitro study. ${ }^{5}$ The lack of PD agreement at these sites may be related to inability to gain good visibility of these surfaces and improper interproximal probing technique. Hassell et al. found that visible areas, like buccal surfaces, and shallow pockets tended to have the most reproducible measurements. ${ }^{7}$ In our study, the majority of PD sites measured between 1 and $4 \mathrm{~mm}$ (96\% of all sites), with $38 \%$ of sites being $2 \mathrm{~mm}$ pockets and $33 \%$ being $3 \mathrm{~mm}$ pockets. This preponderance of relatively shallow sites may be a limitation of this study and may explain why those factors had little effect on PD agreement. Many of the periodontitis patients may have had mild or localized forms of the disease and therefore presented with fewer deep pockets.

We also found no significant difference between the test and control groups for examination time. The faculty examiner took a significantly shorter amount of time to complete the examinations compared to either student group. In fact, the faculty examiner was significantly faster in actual patient examinations than the length of the exam shown in the video, which was eight minutes and 44 seconds long. The goal of the video was to emphasize aspects of proper probing technique to gain more accurate measurements; to achieve this, the examiner in the video moved at a slower pace to allow the viewers time to observe and comprehend the demonstration. The results of the study showed that, compared to the control group, the test group subjects took a longer time to complete their examinations. It can be speculated that these students, after viewing the training video, conducted their examinations more carefully, which resulted in a longer examination time.

When we analyzed PD measurements that exceeded the clinically acceptable variance, we found that all the students had a general tendency for underestimating rather than overestimating their measurements. These findings are consistent with those of two other studies examining general dentists' habits for periodontal referrals. Dockter et al. found that a majority of patients referred to periodontal offices were affected by more severe forms of periodontitis. ${ }^{8}$ Additionally, most patients were treated with non-surgical therapy only, despite differences in disease severity; and many patients were only seen semiannually for maintenance rather 
than the recommended three or four times per year. Their results suggest a trend for referring patients to periodontal offices too late, when the disease has already significantly progressed; these delays may be because of underestimations in diagnosis at an earlier point and attempts to treat less severe forms of the disease. In Lee et al.'s study, 32.6\% of a general dentist population reported seeing an average of zero to five periodontitis patients per week. ${ }^{9}$ Those authors commented that, given the prevalence of periodontal disease in the U.S. population, this average patient count is remarkably low and may be attributed to the general dentists' lack of knowledge of or inability to accurately diagnose periodontitis. This possibility further emphasizes the crucial need to improve students' clinical skills in periodontal diagnosis while in dental school for the benefit of their current and future patients.

The effectiveness of audiovisual recorded demonstrations has previously been studied for operative, ${ }^{10,11}$ prosthodontic, ${ }^{12-14}$ and orthodontic ${ }^{15}$ techniques. Only two studies reported on the efficacy of teaching videos for periodontics. In the first one, Ramlogan et al. examined the effect of an instructional video (filmed by digital camera on a tripod) compared to a live instruction session for several basic periodontal skills, including six-point charting. ${ }^{16}$ Students' level of understanding for these skills was assessed through comparison of written pre- and posttest assessments. The students who received the live lecture scored significantly higher $(74.6 \%)$ than students who watched the instructional video (68.6\%), but $97 \%$ of the students wanted to have the video added to their curriculum. Botticelli et al. also studied the effect of two teaching methods (live lecture involving standard instruction including TVprojected live demonstrations on a mannequin compared to a Visual Training System, which included a presentation with images and video clips) for various skills, including probing and scaling. ${ }^{17}$ The authors evaluated students' performance through video recordings of each subject performing each skill on mannequins. For probing, the examiners evaluated the students' positioning and probe insertion and movements only; the results showed no significant difference between video and live instruction for probing technique.

The use of a camera mounted on loupes provides a distinctive perspective for the viewers: it allows students to see through the instructor's eyes and simulate what their own view inside a patient's mouth may be. With the "Calibrated Periodontal
Training Video," students can see and appreciate how the instructor positions the patient, accesses certain areas of mouth, and views surfaces of particular teeth. Audiovisual tools such as these may be easily disseminated to entire classes through school email, learning management systems, or file hosting services, making access simple and easy. Students may then refer to them at any time for review or study. These benefits overcome some of the disadvantages of live demonstrations, such as the problem of not every student being able to adequately view what is being shown and the non-repeatability of demonstrations. ${ }^{12}$ It should be noted, however, that live demonstrations have been found to allow for immediate student and faculty interaction for any questions, clarifications, or comments. ${ }^{13}$ For this reason in preclinical settings, as suggested by Nikzad et al., it may be preferable to supplement live demonstrations with audiovisual aids, rather than replace them. ${ }^{12}$ Our survey results, as well as results from other studies, showed a student preference for audiovisual tools also. ${ }^{12-14,18,19}$

Our study differed from these previous investigations and had some strengths and limitations. In contrast to those studies, our research was a human randomized controlled trial that examined the effect of video demonstration directly on PD accuracy on live patients in a clinical setting. Another strength was our sample size of 22 students and 70 total patient exams, which exceeded our power calculation. Some students were very enthusiastic and completed more than their required three patient exams. Additionally, we received the maximal response for our anonymous post-intervention survey.

However, the study also had some limitations. First, the subject pool consisted of only third-year students at one dental school, so the results may not be generalizable. A second possible limitation was the result of the decision to not collect any students' demographics or information about their grades or class rank. Students with high class rank will excel clinically, so grades and class standing should be strongly considered when considering clinical outcomes. However, Stewart et al. examined the pass/fail rate of 524 Florida Dental Licensure Exam candidates and found no correlation between mean exam score and quartile rank (based on grade point average) for the periodontics section of the exam. ${ }^{20}$ Third, our PD distribution consisted mainly of 1-4 mm sites only, which restricted our conclusions about deeper PDs of $\geq 5 \mathrm{~mm}$. Future studies with larger sample sizes, more detailed categorization of patient diagnosis, and inclusion of sites with PD $\geq 5$ may provide for 
more definitive conclusions regarding the efficacy of audiovisual tools for improving PD measurement accuracy.

\section{Conclusion}

This study found that using the "Calibrated Periodontal Training Video" improved students" probing depth accuracy for sites with PD of $4 \mathrm{~mm}$ by $10 \%$, but it did not have an effect on examination time or PD accuracy in patients diagnosed with periodontitis. Inexperienced dental students tend to underestimate probing depth measurements. These outcomes illustrate the need for effective teaching methods to demonstrate proper probing technique. Audiovisual aids filmed from the point of view of the examiner on a real patient may help students correlate classroom learning with clinical procedure. The "Calibrated Periodontal Training Video" was well received by these students and may serve as an effective adjunct to current teaching methods. Future studies with larger sample sizes are needed to confirm the effect of this teaching aid on measurement accuracy in sites with $\mathrm{PD} \geq 5 \mathrm{~mm}$. Further investigations of point of view audiovisual tool efficacy may include teaching periodontal skills, such as periodontal instrumentation and scaling and root planing in dental and dental hygiene student cohorts.

\section{Acknowledgments}

This project was funded by the Graduate Research Committee of the IU School of Dentistry.

\section{REFERENCES}

1. Eke PI, Dye BA, Wei L, et al. Update on prevalence of periodontitis in adults in the United States: NHANES 2009 to 2012. J Periodontol 2015;86(5):611-22.

2. Chapple ILC, Mealey BL, Dyke TEV, et al. Periodontal health and gingival diseases and conditions on an intact and a reduced periodontium: consensus report of workgroup 1 of the 2017 world workshop on the classification of periodontal and peri-implant diseases and conditions. J Periodontol 2018;89(Suppl 1):S74-84.

3. Papapanou PN, Sanz M, Buduneli N, et al. Periodontitis: consensus report of workgroup 2 of the 2017 world workshop on the classification of periodontal and peri-implant diseases and conditions. J Periodontol 2018;89(Suppl 1): S173-82.

4. Silva-Boghossian CM, Amaral CSF, Maia LC, et al. Manual and electronic probing of the periodontal attachment level in untreated periodontitis: a systematic review. J Dent 2008;36(8):651-7.
5. Drucker SD, Prieto LE, Kao DWK. Periodontal probing calibration in an academic setting. J Dent Educ 2012;76(11):1466-73.

6. Seabra RC, Costa FO, Costa JE, et al. Impact of clinical experience on the accuracy of probing depth measurements. Quintessence Int Berl Ger 1985 2008;39(7):559-65.

7. Hassell TM, Germann MA, Saxer UP. Periodontal probing: interinvestigator discrepancies and correlations between probing force and recorded depth. Helv Odontol Acta 1973;17(1):38-42.

8. Dockter KM, Williams KB, Bray KS, Cobb CM. Relationship between prereferral periodontal care and periodontal status at time of referral. J Periodontol 2006;77(10): 1708-16.

9. Lee JH, Bennett DE, Richards PS, Inglehart MR. Periodontal referral patterns of general dentists: lessons for dental education. J Dent Educ 2009;73(2):199-210.

10. Patel SA, Barros JA, Clark CM, et al. Impact of techniquespecific operative videos on first-year dental students performance of restorative procedures. J Dent Educ 2015; 79(9):1101-7.

11. Gadbury-Amyot CC, Purk JH, Williams BJ, Ness CJV. Using tablet technology and instructional videos to enhance preclinical dental laboratory learning. J Dent Educ 2014;78(2):250-8.

12. Nikzad S, Azari A, Mahgoli H, Akhoundi N. Effect of a procedural video $\mathrm{CD}$ and study guide on the practical fixed prosthodontic performance of Iranian dental students. J Dent Educ 2012;76(3):354-9.

13. Packer ME, Rogers JO, Coward TJ, et al. A comparison between videotaped and live demonstrations for the teaching of removable partial denture procedures. Eur J Dent Educ 2001;5(1):17-22.

14. Aragon CE, Zibrowski EM. Does exposure to a procedural video enhance preclinical dental student performance in fixed prosthodontics? J Dent Educ 2008;72(1):67-71.

15. Alqahtani ND, Al-Jewair T, AL-Moammar K, et al. Live demonstration versus procedural video: a comparison of two methods for teaching an orthodontic laboratory procedure. BMC Med Educ 2015;15:199.

16. Ramlogan S, Raman V, Sweet J. A comparison of two forms of teaching instruction: video vs. live lecture for education in clinical periodontology. Eur J Dent Educ 2014;18(1):31-8.

17. Botticelli AT, Janda MS, Botticelli D, et al. The effectiveness of video support in the teaching of manual skills related to initial periodontal therapy tested on phantoms. Int J Comput Dent 2005;8:117-27.

18. Fakhry A, Cooper S, Slach N, Krenz S. Video-assisted clinical instruction in dentistry: overview and applications. Eur J Dent Educ 2007;11(4):230-7.

19. Rystedt H, Reit C, Johansson E, Lindwall O. Seeing through the dentist's eyes: video-based clinical demonstrations in preclinical dental training. J Dent Educ 2013;77(12):1629-38.

20. Stewart CM, Bates RE, Smith GE. Relationship between performance in dental school and performance on a dental licensure examination: an eight-year study. J Dent Educ 2005;69(8):864-9. 